Bull. Mater. Sci., Vol. 6, No. 1, February 1984, pp. 1-5.

(C) Printed in India.

\title{
Ferromagnetic resonance in metallic glasses: study of fracture, stress and thermal stability
}

\author{
R S PARASHAR, C S SUNANDANA AND ANIL K BHATNAGAR \\ School of Physics, University of Hyderabad, Hyderabad 500134 , India

\begin{abstract}
Effects of fracture, stress and isothermal annealing of $\mathrm{Fe}-\mathrm{Ni}$ based metallic glasses have been investigated using the ferromagnetic resonance technique. FMR linewidth is quite sensitive to changes in the magnetic and structural order in metallic glasses, and FMR lineshape seems to provide useful qualitative information on the mechanical state of these systems. Our observations are compared with recent work of Baianu and co-workers.
\end{abstract}

Keywords. Ferromagnetic resonance; metallic glasses; fracture; stress; thermal stability.

\section{Introduction}

Mechanical and thermal stability are two important aspects in the studies of metallic glasses from theoretical as well as practical points of view. These alloys are distinguished by their exceptional strength and distinctive deformation mechanism (Davis 1976). Magnetic properties of ferromagnetic metallic glasses and the influence of annealing on these properties have received much attention in recent years in view of their potential technological applications (Lubrosky et al 1978). Among many other techniques to study the effects of thermal and mechanical treatments on the magnetic and mechanical properties of metallic glasses, ferromagnetic resonance (FMR) is a convenient and very sensitive technique (Baianu et al 1979a, b) since FMR linewidths and lineshapes are very sensitive to the magnetic and mechanical states of the system. In this paper our preliminary results of such studies on $\mathrm{Fe}_{40} \mathrm{Ni}_{40} \mathrm{P}_{14} \mathrm{~B}_{6}$ (Metglas 2826), $\mathrm{Fe}_{29} \mathrm{Ni}_{49} \mathrm{P}_{14} \mathrm{~B}_{6} \mathrm{Si}_{2}$ (Metglas 2826B) and $\mathrm{Fe}_{40} \mathrm{Ni}_{40} \mathrm{~B}_{20}$ (vitrovac 0040) are reported.

\section{Experimental}

Commercially available ferromagnetic metallic glasses $\mathrm{Fe}_{40} \mathrm{Ni}_{40} \mathrm{P}_{14} \mathrm{~B}_{6}$ and $\mathrm{Fe}_{29} \mathrm{Ni}_{49} \mathrm{P}_{14} \mathrm{~B}_{6} \mathrm{Si}_{2}$ were received from Allied Chemical Corp. (USA) and $\mathrm{Fe}_{40} \mathrm{Ni}_{40} \mathrm{~B}_{20}$ was received from Vacuumschmelze (West Germany) in thin ribbon forms. Fractured specimens were prepared by cutting/fracturing by (1) bending on a sharp surgical knife edge by $180^{\circ}$ (method A), (2) use of a sharp pair of scissors (method B), and (3) a rotating diamond wheel (method C). All the specimens were cut perpendicular to the long ribbon axis. For annealing studies, samples were first polished by 600 grade emery paper and then isothermally annealed at different temperatures and for different times.

First derivative FMR spectra were recorded on a X-band $(9.3 \mathrm{GHz})$ JEOL ESR spectrometer (model FE-3X) using approximately $1 \mathrm{~mW}$ incident radiation power. Specimens were mounted such that the plane of each specimen and its long ribbon axis were always parallel and perpendicular to the applied magnetic field, respectively. For isothermally treated specimens only a small (approximately $1 \mathrm{~mm}$ diameter) central 
portion of the ribbon was exposed to the incident microwaves to minimize demagnetization effects. The observed peak-to-peak FMR linewidth $\left(\Delta H_{p p}\right)$ of the polished ribbon was approximately $235 \mathrm{Oe}$.

\section{Results and discussion}

\subsection{Fracture study}

Amorphous metallic ribbons, when deformed at temperatures much less than the glass transition temperature, develop highly inhomogeneous shear bands. The failure occurs instantaneously at the yield stress by'shear rupture through an intense shear band. Propagation of the shear band on an excess volume front gives rise to distinct surface features depending upon the deformation method used, since the distribution of forces exerted by different fracturing methods would be different. So far, fracture studies in these alloys seem to have been limited only to the optical and electron microscopic investigations. In FMR, the magnetization variation at the boundaries as well as surface pits, grain boundaries, and disordered scattering all make contributions to the intrinsic lineshape and linewidth. Hence, FMR studies along with those of scanning electron microscope (SEM) on these alloys could give more detailed information on the magnetic state of the fractured boundaries. SEM observations have shown that fracturing of a metallic glass ribbon by methods $A$ and $B$ give rise to highly inhomogeneous surface boundaries while method $C$ gives rise to the least deformation (Parashar et al 1982). The width of the shear lines are observed to vary in the range $0 \cdot 8-2 \mu \mathrm{m}$. FMR results on a Metglas 2826B alloy specimen cut using method A show distorted lineshape, additional satellites and a few extra resonance peaks as shown in figure la. We have observed that such effects are considerably reduced in the scissors-cut specimens, as shown in figure $1 \mathrm{~b}$, indicating it to be a comparatively better of way of cutting of a metallic glass which gives rise to less mechanical damage at the surface boundary, and less fluctuations in the height of shear markings. Removal of such deformation would result in a smoother surface which would in turn reduce the linewidth. This was done by electropolishing of the specimen. FMR result on a typical electropolished sample is shown in figure 1c. A comparison of FMR spectra in figure 1 shows that the electropolished specimens give smooth and narrow resonance curves. The present results are in qualitative agreement with those reported earlier by Baianu et al $(1979 \mathrm{a})$. In the case of the diamond wheel cut specimen it was observed that the linewidth changes before and after electropolishing the specimen were minimal indicating that this cutting method, method $\mathrm{C}$, induces least deformation in the specimen.

\subsection{Effect of stress annealing}

It is well-known that the application of a tensile stress tends to align magnetic domains in ferromagnetic amorphous alloys along the direction of the applied stress. The resistivity has been observed to show a linear variation with the applied stress and the slope of the curve depends upon the sign of the magnetostriction coefficient of the amorphous alloy. This behaviour of magnetic metallic glasses is similar to that observed for ferromagnetic crystalline metals. It has been observed that $\mathrm{Fe}_{49} \mathrm{Ni}_{29} \mathrm{P}_{14} \mathrm{~B}_{6} \mathrm{Si}_{2}$, when annealed for $20 \mathrm{hr}$ at $450 \mathrm{~K}$, still shows amorphous structure when examined by $x$-ray diffraction measurements. However, we have observed that 


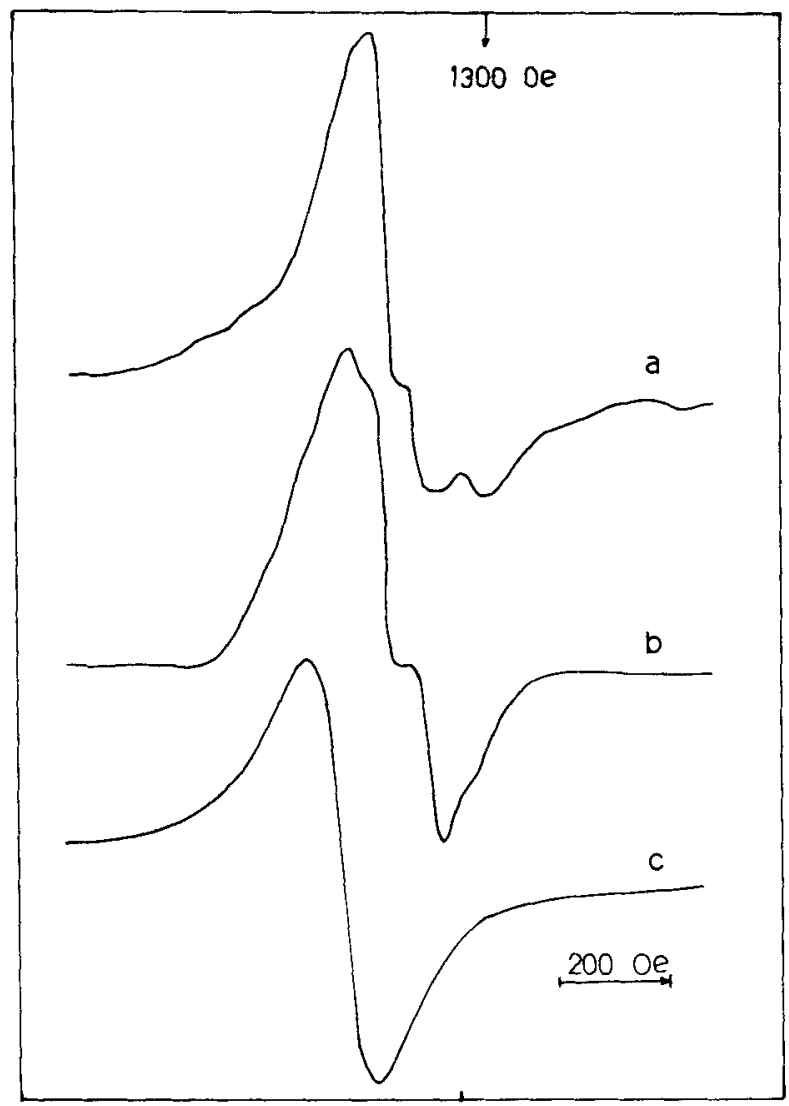

Figure 1. FMR spectra of $\mathrm{Ni}_{40} \mathrm{Fe}_{40} \mathrm{~B}_{14} \mathrm{P}_{6}$ amorphous alloy ribbons fractured by (a) bending, (b) using scissors, and (c) scissors, and electropolished subsequently.

samples stress-annealed (temperature varied at the rate of $3 \mathrm{~K} / \mathrm{min}$ upto $440 \mathrm{~K}$ and a load of $9.8 \mathrm{~kg} / \mathrm{mm}^{2}$ ) showed some precipitated crystalline phase (Parashar and Bhatnagar 1983). Stress-annealed samples have also found to exhibit broad FMR peaks whose linewidths are larger when compared with stress free annealed samples under similar experimental conditions (Parashar and Bhatnagar 1983).

\subsection{Isothermal annealing study}

In view of their metastable equilibrium configuration resulting from rapid quenching, metallic glasses are thermodynamically unstable with respect to both crystalline and fully-relaxed amorphous states. Thus structural relaxations can be induced in these alloys by annealing them at temperatures considerably below their crystallization temperatures. Although amorphous alloys possess neither long-range order nor vacancies, it is nevertheless likely that the structural changes occurring during relaxation involve both the amount and distribution of free volume and the nature of local atomic arrangement. However, they do possess extensive short-range order (quite akin to their crystalline counterparts) which is strongly temperature-dependent. Annealing decreases the fictive temperature (the temperature at which the structure 
would be in equilibrium with the undercooled liquid) and further increases the shortrange order until metastable equilibrium is attained at the annealing temperature. This relaxation, involving collective motion of atoms and exchange of atoms of different chemical species, results in changes of variety of physical properties e.g. Curie temperature, thermal expansion coefficient and magnetic amisotropy etc. We have used FMR linewidth as a probe to study the effect of isothermal annealing in $\mathrm{Fe}_{40} \mathrm{Ni}_{40} \mathrm{~B}_{20}$ ribbons.

Figure 2 gives the typical variation of $\Delta H_{p p}$ with annealing time, for sample annealed at $100^{\circ} \mathrm{C}$ which is substantially lower than the crystallization temperature $\left(\sim 400^{\circ} \mathrm{C}\right)$ for $\mathrm{Fe}_{40} \mathrm{Ni}_{40} \mathrm{~B}_{20}$ glassy alloy. Two maxima, one around $1 \mathrm{~min}$ and the other $10 \mathrm{~min}$, are clearly seen. These peaks, which seem to characterize the short-term and long-term annealing behaviour of this alloy, need to be accounted for in terms of structural and magnetic ordering influenced by annealing.

Baianu et al (1979b) in their FMR study on $\mathrm{Fe}_{40} \mathrm{Ni}_{40} \mathrm{P}_{14} \mathrm{~B}_{6}$ ribbons have observed a rather different time-dependence for samples annealed at $350^{\circ} \mathrm{C}$. The linewidth decreases rapidly at first and slowly later upto annealing periods of 60 minutes and increases thereafter. They have attributed this linewidth narrowing due to magnetic ordering of magnetic dipoles in a matrix involving phosphorous rich regions and with the stress relief within the sample. However, in the present amorphous alloy, annealing at lower temperature $\left(100^{\circ} \mathrm{C}\right)$ which causes an increase in linewidth, may be associated with changes in the magnetic atom neighbourhood, by way of local structural regrouping and enhancement of atomic structural inhomogeneity through cluster formation. Mössbauer effect studies indeed show the clustering of $\mathrm{Fe}-\mathrm{Ni}$ atoms in annealed $\mathrm{Fe}_{40} \mathrm{Ni}_{40} \mathrm{~B}_{20}$ samples (Vincze and Babic 1978). Decrease in electrical resistivity may also be a possible cause of an increase in the linewidth (Baianu et al 1979a). However, resistivity changes observed by Stubicar et al (1977) in $\mathrm{Fe}_{\mathbf{4 0}} \mathrm{Ni}_{\mathbf{4 0}} \mathbf{B}_{\mathbf{2 0}}$ are too small to be correlated with the linewidth changes observed here; therefore, the reason for an increase in linewidth of $\mathrm{FMR}$ of $\mathrm{Fe}_{40} \mathrm{Ni}_{40} \mathrm{~B}_{20}$ may be due to other reasons like clustering etc.

Recent studies of time-dependence of Young's modulus during isothermal annealing, employing ultrasonic techniques, on $\mathrm{Ni}_{40} \mathrm{Fe}_{40} \mathrm{~B}_{20}$ ribbons (Scott and $\mathrm{Kursumovic}$

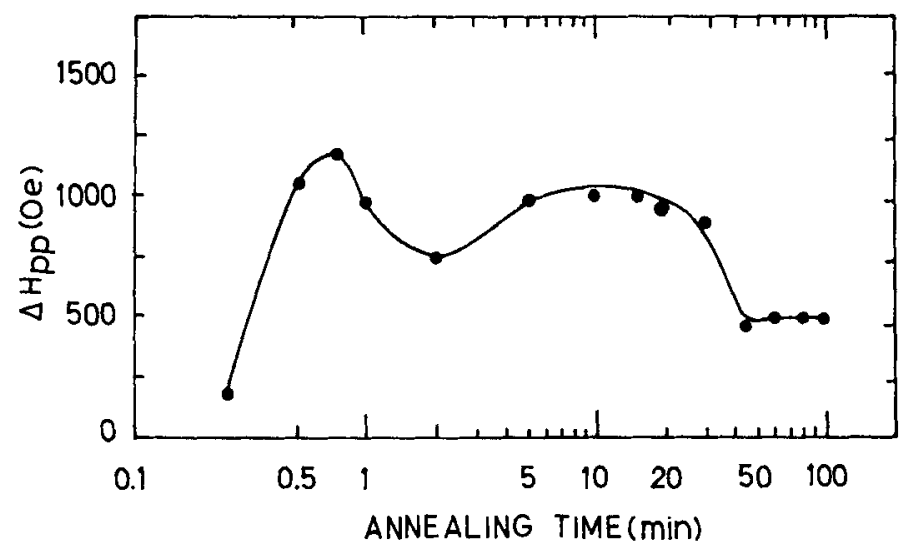

Figure 2. Dependence of $\mathrm{FMR}$ linewidth on annealing time for $\mathrm{Ni}_{40} \mathrm{Fe}_{40} \mathrm{~B}_{20}$ amorphous alloy ribbons isothermally annealed at $100^{\circ} \mathrm{C}$. 
1982) and dilatometric studies on the same sample (Kursumovic et al 1980) give additional insight into the local atomic rearrangement involving quenched-in defects when they are subjected to isothermal annealing. These defects are nothing but local structural deviations from ideal glass by way of distinctly different metalloid polyhedra around magnetic alloy and annealing causes these defects to relax and their number and mobility decrease as a consequence.

The study by Scott and Kursumovic (1982), Kursumovic et al (1980) and Greer and Leake (1979) are 'crossover experiments' in which plots of ultrasonic velocity, dilatation and $T_{c}$ against annealing time exhibit a minimum for annealing times of the order of a minute. This 'crossover' has been described as a relaxation process involving at least two relaxation times. On the other hand the plot of thermal expansion of Metglas 2826 and Metglas $2826 \mathrm{~B}$ ribbons against annealing time shows a maximum in annealing time $\left(\sim 5 \mathrm{~min}\right.$ ) when annealed at $250^{\circ} \mathrm{C}$, which has been attributed to initial rapid stress relaxation' (Steinberg et al 1980). Potocky et al (1982) have recently investigated the dependence of coercive force on the annealing time on amorphous $F_{60} \mathbf{N i}_{20} \mathbf{B}_{20}$ ribbons isothermally annealed at temperature well below crystallization temperature. They find that the relaxation of 'quenched-in' stress upon annealing takes place in two stages, the first involving the stress centres and the other involving topological changes. In the light of these observations, the twin maxima in the FMR linewidth vs annealing time plot observed in the present study could be reasonably attributed to relaxation processes involving two magnetically and chemically distinct species. However, a quantitative characterization of relaxation times and the species involved needs theoretical models to be developed which must take into account the nature, number and distribution of metalloid neighbourhoods as well as the magnetic interaction among them. Further more extensive work is continuing to understand this behaviour.

\section{Acknowledgement}

The work has been supported by the Science and Engineering Research Council (DST, India).

\section{References}

Baianu I C, Patterson J and Rubinson K A 1979a Mater. Sci. Engg. 40273

Baianu I C, Rubinson K A and Patterson J 1979b J. Phys. Chem. Solids 40941

Davis L A 1976 in Rapidly quenched metals, (eds) N J Grant and B C Giessen (MIT: Cambridge, USA) vol. 1, p. 369

Greer A L and Leake J A 1970 J. Noncrystalline Solids 33291

Kursumovic A, Scott M G and Cahn R W 1980 Scr. Metall. 141245

Luborsky F E, Frischmann P G and Johnson L A 1978 J. Magn. Magnetic Mater. 8318

Parashar R S, Sunandana C S and Bhatnagar A K 1982 Preprint

Parashar R S and Bhatnagar A K 1983 J. Magn. Magnetic Mater. 3656

Potocky L, Zentko A, Novak L and Svidron V 1982 J. Magn. Magnetic Mater. 26115

Scott M G and Kursumovic A 1982 Acta Metall. 30853

Steinberg J, Tyagi S and Lord Jr A E 1980 J. Noncrystalline Solids 41279

Stubicar M, Babic E, Subasic D, Pavuna D and Marohnic Z 1977 Phys. Status. Solidi A44 339

Vincze I and Babic E 1978 Solid State Commun. 271425 